\title{
Chatbot como herramienta comunicativa durante la crisis sanitaria de la COVID-19 en España
}

\section{Chatbot as a communication tool during the COVID-19 health crisis in Spain}

\section{Chatbot como ferramenta de comunicação durante a crise de saúde COVID-19 na Espanha}

\author{
Gema Bonales $^{1}$ \\ Universidad Complutense de Madrid (España) \\ gbonales@ucm.es \\ Nuria Pradilla ${ }^{2}$ \\ Universidad Complutense de Madrid (España) \\ npradill@ucm.es \\ Eva Citlali ${ }^{3}$ \\ Universidad Complutense de Madrid (España) \\ evacmart@ucm.es
}

Fecha de recepción: 27 de noviembre de 2020

Fecha de recepción evaluador: 4 de diciembre de 2020

Fecha de recepción corrección: 13 de diciembre de 2020

\footnotetext{
${ }^{1}$ Profesora Asociada en el Departamento de Ciencias de la Comunicación Aplicada. ORCID: https://orcid.org/00000003-2085-2203

${ }^{2}$ Doctora en Comunicación Audiovisual y profesora asociada en el Departamento de Diseño e Imagen (Dibujo II) de la Facultad de Bellas Artes. ORCID: https://orcid.org/0000-0001-6566-7744

${ }^{3}$ Doctoranda en Comunicación Audiovisual y Publicidad y Máster en Comunicación de las Organizaciones. ORCID: https://orcid.org/0000-0001-6599-1050
} 


\title{
Resumen
}

La expansión de la Covid-19 a nivel mundial desembocó en un colapso de las redes sanitarias en la mayoría de los países. En esta situación, las nuevas tecnologías adquirieron un papel relevante, especialmente en la gestión de la comunicación, gracias a su inmediatez y posibilidades de cobertura. El confinamiento establecido en España, como consecuencia del estado de alarma decretado en marzo de 2020, aceleró la adopción de diversos recursos tecnológicos en el área de la atención médica a distancia. En este contexto, las plataformas conversacionales se han ido convirtiendo en una de las herramientas más utilizadas por las instituciones para aliviar la falta de recursos en atención telefónica y presencial a la hora de atender las primeras consultas realizadas por los ciudadanos aquejados de síntomas, o por aquellos que solicitaban algún otro tipo de información urgente relacionada con la enfermedad. Esta demanda de agilidad vino a ser atendida por diferentes empresas que ofrecieron el desarrollo de servicios de bot en tiempo record. "Los chatbots son una solución que permite de forma rápida implementar un medio de respuesta inmediato a los usuarios" (Aguilar et al., 2020). El presente artículo analiza el grado de alcance que estas plataformas de respuesta en línea tuvieron -y aún tienen- en la descongestión de los servicios sanitarios en España y si su disponibilidad ha sido de algún modo útil para los ciudadanos. Para ello, se ha tomado una muestra de las cinco principales plataformas conversacionales y, utilizando un estudio de caso, se han revisado sus características particulares. Además se han aportado los datos obtenidos a través de un cuestionario realizado a usuarios y los extraídos de una serie de entrevistas a expertos. El resultado de esta investigación pone de manifiesto que el nivel de uso de los chatbots sanitarios específicos para la Covid-19 en la actualidad alcanza al 30\% de la muestra analizada y que los que han empleado este tipo de asistentes lo han hecho fundamentalmente con fines informativos, siendo los meses de abril y octubre los que han reflejado mayor actividad en este sentido.

Palabras clave: Comunicación; Tecnología; Chatbot; Humano-máquina; Covid-19

\begin{abstract}
The spread of Covid-19 worldwide led to a collapse of the health networks in the vast majority of western countries. In this situation, the new information technologies acquired a relevant role, especially in communication management, thanks to their ubiquity, immediacy and coverage possibilities. The confinement established in Spain, as a consequence of the state of alarm decreed in March 2020, accelerated the adoption of various technological resources in the area of remote medical care. In this context, the conversational platforms have become one of the most used tools by institutions to alleviate the scarcity of resources in telephone and face-to-face attention when attending the first consultations made by patients suffering from symptoms, or by those who requested some other type of urgent information related to the disease. This demand for agility was be met by different companies that offered the development of bot services in a very short time. "Chatbots are a solution that allows to implement quickly a way of immediate response for users" (Aguilar et al., 2020). This article analyses the grade of
\end{abstract}


reach that these online response platforms had -and are still having-in decongesting the health services in Spain and how their availability has been useful for the citizens. For this, a sample of the five main conversational platforms has been taken and, using a case study, their particular characteristics have been reviewed. In addition, the data obtained through a questionnaire made to users and a series of interviews with experts have been taken into account and studied. The result of this research shows that the level of use of specific health chatbots for Covid-19 currently reaches $30 \%$ of the analysed sample and that those who have used this type of assistants have done so mainly for informative purposes. The months of April and October reflected the most activity in this sense.

Keywords: Communication; Technology; Chatbot; Human-machine; Covid-19

\section{Resumo}

A disseminação da Covid-19 em todo o mundo levou ao colapso das redes de saúde na maioria dos países. Nesta situação, as novas tecnologias adquiriram um papel relevante, sobretudo na gestão da comunicação, pela sua rapidez e possibilidades de abrangência.

O confinamento estabelecido em Espanha, na sequência do estado de alarme decretado em março de 2020, acelerou a adoção de diversos recursos tecnológicos na área da assistência médica à distância. Nesse contexto, as plataformas de conversação têm-se tornado uma das ferramentas mais utilizadas pelas instituições para amenizar a escassez de recursos no atendimento telefónico e presencial no atendimento às primeiras consultas feitas por cidadãos com sintomas, ou por aqueles que solicitaram algum outro tipo de informação urgente relacionada com a doença. Essa procura por agilidade passou a ser atendida por diversas empresas que ofereciam o desenvolvimento de serviços de bot em tempo recorde. "Os chatbots são uma solução que permite implementar rapidamente um meio de resposta imediata aos utilizadores" (Aguilar et al., 2020). Este artigo analisa o grau de abrangência que essas plataformas de resposta online tiveram -e ainda têm- no descongestionamento dos serviços de saúde na Espanha e se a sua disponibilidade tem sido útil de alguma forma para os cidadãos. Para isso, foi feita uma amostra das cinco principais plataformas de conversação e, por meio de um estudo de caso, as suas características particulares foram revistas. Além disso, foram disponibilizados os dados obtidos através de um questionário dirigido aos usuários e também de uma série de entrevistas com especialistas. O resultado desta pesquisa mostra que o nível de uso de chatbots de saúde específicos para Covid-19 atualmente atinge 30\% da amostra analisada e que aqueles que usaram este tipo de assistentes o fizeram principalmente para fins informativos, sendo os meses de abril e outubro os que mais refletiram atividade neste sentido.

Palavras-chave: Comunicação; Tecnologia; Chatbot; Homem-máquina; Covid-19 


\section{Introducción}

Debido al alarmante nivel de propagación del SARS-CoV-2 a escala mundial y la imposibilidad de controlar la enfermedad, el 11 de marzo de 2020 el Director General de la Organización Mundial de la Salud (OMS) declaró en rueda de prensa que se estaba, por primera vez en la historia, ante una pandemia causada por un coronavirus (2020). Tres días después, el Gobierno de España anunció el decreto por el que se declaraba el estado de alarma para hacer frente a la expansión del virus. Esta medida, que entró en vigor el 15 de marzo, dio inicio a una etapa de confinamiento que se prolongó durante 99 días. Este hecho insólito ocasionó la prohibición del libre movimiento de las personas en todo el territorio a excepción de una serie de desplazamientos considerados esenciales.

Las cifras de expansión de la epidemia tuvieron su punto más álgido el 26 de marzo cuando se registraron 9.181 contagios mientras el número de muertes diarias atribuidas al coronavirus parecían no tocar techo (Flores, 2020). Como consecuencia, numerosas iniciativas públicas y privadas han desarrollado aplicaciones y bots específicos relacionados con el coronavirus para evitar el colapso sanitario y de sus redes de comunicación. La propia Organización Mundial de la Salud lanzó el 25 de marzo de 2020 un chatbot en inglés que tendría su versión en español cinco días después. Estas plataformas conversacionales han sido una pieza clave porque han proporcionado información, seguimiento, monitoreo y control sanitario al ciudadano en una primera fase de la detección de la enfermedad, especialmente cuando el sistema sanitario estaba saturado con enfermos y personas que presentaban síntomas compatibles con la enfermedad (Miner et al., 2020 y Ahuja et al., 2020).

Desafortunadamente, en la fecha de publicación de este estudio, la pandemia está lejos de ser erradicada y la rápida expansión de contagios observada durante la segunda ola amenaza de nuevo al sistema sanitario y, por este motivo, el 25 de octubre se aplicó en España un segundo estado de alarma, destinado, en este caso, a proveer a las comunidades autónomas de las herramientas legales necesarias para establecer las medidas que consideren oportunas para frenar los contagios; como los cierres perimetrales, cierre de comercios, toques de queda, etc.

Este trabajo tiene como propósito estudiar el papel de los bots en esta crisis en cuanto a herramienta de comunicación. Se trata de observar si este tipo de plataformas cumplen los objetivos planteados en su diseño, si se han demostrado como aplicaciones válidas desde el punto de vista de la experiencia de los usuarios y de conocer cómo han contribuido a las estrategias de comunicación estatales y de otras instituciones en el contexto de la pandemia por Covid-19. 


\section{Marco referencial}

\section{Comunicación entre el hombre y la tecnología}

Al hablar del tema humano- máquina, en especial de la Inteligencia Artificial, es importante tener en cuenta la Teoría del Valle Inquietante, la cual asegura que el grado de preferencia del ser humano por una inteligencia artificial dependerá de si esta se vuelve más humana. En caso de no ser así, la inclinación por ella disminuye. Hay una gran afinidad de los sistemas que utilizan avatares con formas humanas, porque se facilita que el usuario se identifique con el desarrollo tecnológico en cuestión (Mori, 1970; Wilks, 2010).

Los bots, según Ferrara (2016), son robots informáticos que operan de manera online y remota. Sin embargo, atendiendo a la función concreta para la que hayan sido diseñados, adoptan uno u otro nombre y el término chatbot alude a un "sistema de conversación de máquinas que interactúan con usuarios humanos a través de un lenguaje de conversación natural" (Shawar y Atwell, 2005, p. 489).

Desde la creación de las aplicaciones de interfaz hombre-computadora, el uso de claves sociales en la interacción con la computadora ha tenido un desarrollo continuo (Prendinger y Shizuka, 2004). En ese sentido, es importante profundizar en qué funciones ejercen los bots y los asistentes de voz en los actos o procesos de comunicación en un periodo tan grave como el actual. Las máquinas inteligentes tienen unos programas de cómputo apropiados para resolver unos problemas y no otros y aunque utilizan los elementos sintácticos de la lengua no usan los semánticos (Malpica, 2016, p.9), y es ahí donde radica su principal dificultad para su comunicación con el hombre y para lograr imitarlo al cien por cien. Sus algoritmos son lógicos, no humanos, no forman parte de un proceso de razonamiento consciente de la máquina, ya que la consciencia es la capacidad de debatir, reflexionar y darse cuenta de su propia existencia que tiene el humano.

En los últimos años, los estudios sobre comunicación y tecnología se han centrado en buscar formas más avanzadas para medir la utilidad y efectividad resultante de la interacción entre el hombre y la computadora, y, al mismo tiempo, conocer cómo es la respuesta del usuario. A través de diferentes estudios como los de Lind y Salomonson (2006), Brandtzaeg y Folstad, (2017), Van den Broeck et al. (2019), entre otros investigadores, se ha comprobado que los robots conversacionales pueden llegar a mejorar la experiencia de los usuarios en línea, especialmente, porque potencian en ellos la sensación de estar siendo atendidos en el momento preciso en el que lo necesitan. Aunque, en la actualidad, es difícil analizar el grado de interacción que existe entre estos asistentes y los consumidores y el nivel de comprensión real que tienen los usuarios de las informaciones proporcionadas por este medio.

Una de las ventajas principales de las plataformas conversacionales es que pueden ser utilizadas por diferentes organizaciones para la comunicación individual con el consumidor, alcanzando -a pesar de ser un proceso automatizado- unos niveles de personalización e interacción óptimos (Van den Broeck et al., 2019 y Kunse, 2016). Esto 
es posible gracias a que los chatbots están diseñados específicamente para ser capaces de mantener conversaciones altamente personalizadas con los usuarios, utilizando esquemas de diálogo pre-programados donde el cliente puede hacer uso de un lenguaje natural o utilizar ciertos botones con opciones propuestas y así obtener recomendaciones particulares e información relevante.

El papel de la tecnología en situaciones de crisis se ha demostrado como fundamental y más aún cuando esta afecta a la salud a un nivel global, como sucede actualmente. Los desarrollos tecnológicos en el área de las TICs y, en especial, la implementación de los bots pueden colaborar de manera importante a mejorar la atención sanitaria y a optimizar sus recursos, resolviendo problemas de comunicación e información y mitigando otros de índole diversa entre los que podríamos incluir los psicológicos o los derivados del aislamiento. Sin embargo, al realizar una revisión bibliográfica para este estudio, se ha observado que no existen investigaciones centradas en la efectividad y el alcance real de estas aplicaciones (Zarouali et al., 2018, Brandtzaeg y Folstad, 2017). Tampoco se han hallado estudios que relacionen el papel de las plataformas conversacionales en situaciones de emergencia sanitaria y colapso de los sistemas de salud. En consecuencia, parece del todo pertinente que la experiencia adquirida en esta situación extrema sirva para estimular el desarrollo de estudios relacionados con la evolución de la tecnología digital en estas situaciones, y, en concreto, con el uso de los chatbots en el contexto de la pandemia de la Covid-19, lo que será de gran utilidad para el desarrollo y mejora de herramientas relacionadas con los procesos de comunicación entre hombres y máquinas.

La interacción entre el usuario y el bot se enmarca dentro de los géneros conversacionales, basándose en la comunicación directa con la audiencia mediante el diálogo (Trillo-Domínguez et al., 2017). Gracias a esta conexión se busca establecer una relación de confianza para poder responder a sus preferencias e inquietudes. Estos medios conversacionales dominan la retórica digital a través de un lenguaje natural, que debe ser lo más parecido al de un ser humano: "el potencial de los bots, está en su distribución personalizada y en su poder conversador con las audiencias, mediante aplicaciones de mensajería instantánea" (Sánchez-González et al., 2017, p. 67).

Los chatbots incorporan cuatro características fundamentales que detallan su forma de comportamiento: la capacidad de actuar de forma autónoma, la automatización total o parcial, requieren de una orientación inicial y se utilizan a través de los medios de comunicación en línea (Grimme et al., 2017, Hofeditz et al., 2020). Además, su sistema de funcionamiento es muy sencillo y cualquier persona está capacitada para manejarlo ya que el programa está preparado para responder a sus preguntas (Dahiya, 2017). En general, parte de su atractivo reside en que estos bots están disponibles instantáneamente las 24 horas del día, los 7 días de la semana. El chatbot puede hacer que la información esté habilitada a una escala mayor y también mejora el servicio al cliente, porque proporciona una rápida respuesta al interesado (Van den Broeck et al., 2019 y Herriman et al., 2020). 
Los investigadores Miner, Laranjo y Kocaballi (2020) opinan que las pandemias tienen tres características únicas que las hacen susceptibles de intervenciones personalizadas a través de estos robots. En primer lugar, las acciones individuales pueden empeorar significativamente los resultados en estas crisis, ya que un solo individuo puede infectar a más personas, dependiendo de su comportamiento. En segundo lugar, existe un miedo al contagio y surge un "estigma relacionado con la enfermedad, como resultado, las personas pueden sentirse personalmente responsables de los malos resultados durante una pandemia y también ocultar síntomas" (Miner, Laranjo y Kocaballi, 2020). La tercera característica se refiere a que durante una situación de este tipo se deben evitar las reuniones físicas de muchas personas, lo cual puede empeorar el riesgo de futuros problemas de salud mental.

En este contexto, se puede decir que los bots evitan el congestionamiento de las redes de comunicación e instalaciones del sistema de salud, especialmente de los centros de atención, y, en consecuencia, contribuyen a minimizar el riesgo de contagio por contacto. Además, el desarrollo de estas plataformas basadas en la inteligencia artificial, también puede colaborar en la educación sanitaria de la población, por ejemplo, reduciendo la posibilidad de que se expandan informaciones falsas en torno a la Covid19. Del mismo modo, la presencia de este tipo de tecnología, ayuda a las instituciones a tener un acercamiento más amigable y cercano con los ciudadanos preocupados (Ahuja et al., 2020). Por tanto, los robots conversacionales tienen posibilidades únicas para fortalecer el bienestar general, porque pueden mitigar el peso emocional que ocasionan las enfermedades infecciosas a corto y largo plazo en las pandemias y así, impulsar una comunicación activa entre las autoridades o responsables sanitarios con cada individuo.

Cuando un usuario introduce cualquier pregunta en un bot, el programa utiliza la inteligencia artificial (IA) para dar la respuesta de inmediato, presentando el texto en forma conversacional. Esto, a su vez, facilita la colaboración con los investigadores y los centros de ayuda, porque gracias al conjunto de información recabada en las conversaciones entre humano-computador, se puede tener un alto nivel de automatización y enriquecer los bancos de datos. 
Figura 1. Funcionamiento chatbot: Flujo conversacional (conversational flow).

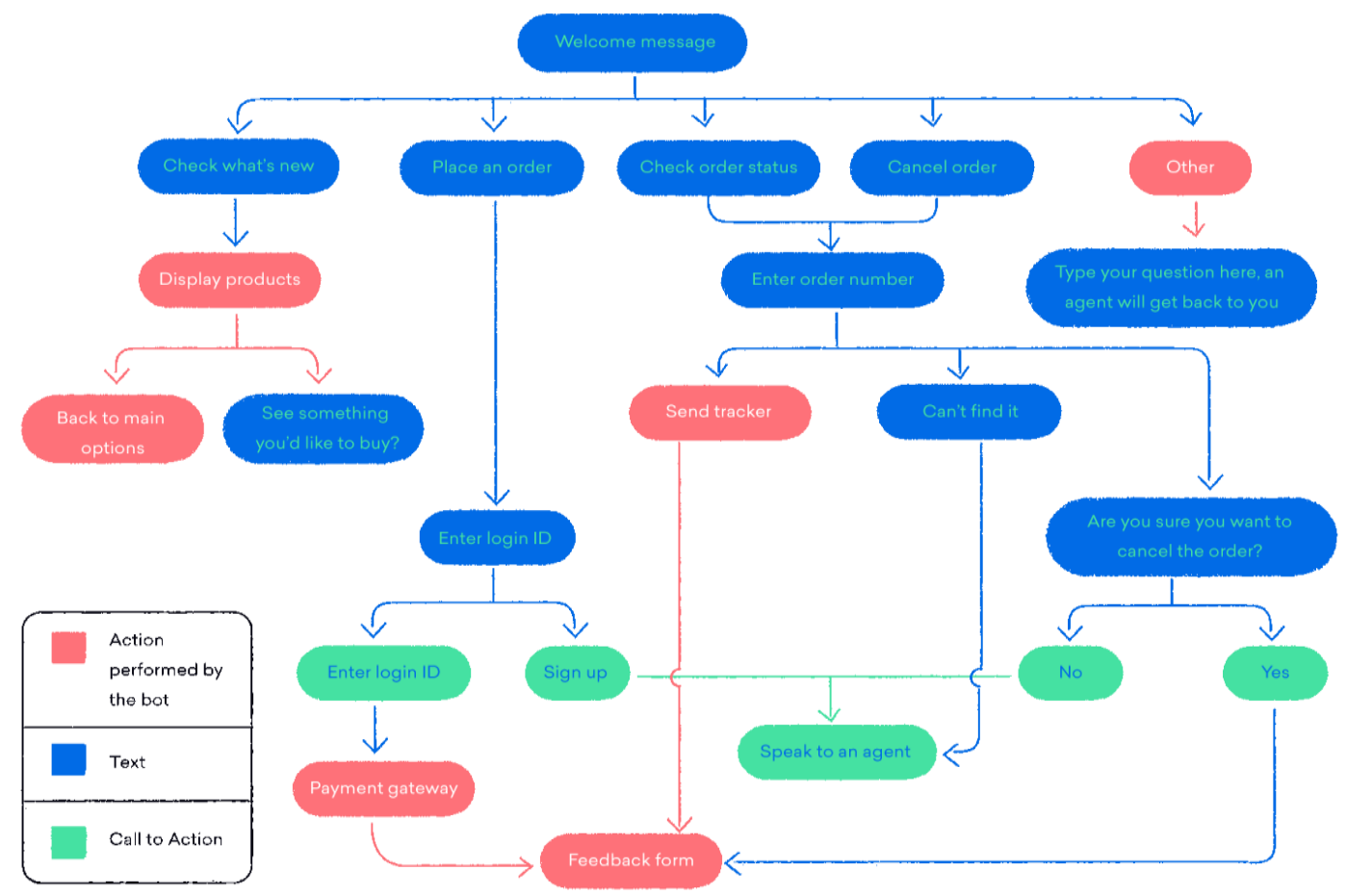

Fuente: Freshdesk, 2020.

El flujo conversacional de los chatbots diseñados a partir de un modelo lingüístico, como el que refleja la Figura 1, está basado en un diálogo lineal estructurado en bloques. Estos diagramas siempre comienzan con un mensaje de bienvenida (wellcome message) y una respuesta predeterminada (answer default). Sin embargo, como se puede observar, las respuestas que proporcionan se limitan a contestar a preguntas frecuentes (FAQs). La posibilidad de generar respuestas más inteligentes y versátiles y que se salieran de ese guión preestablecido requerirían de una actualización constante. Este aprendizaje automático lo consiguen los robots que utilizan IA en su diseño, requiriendo, por tanto, una mayor inversión. También existen modelos híbridos, que tienen como ventajas el que permiten que las soluciones se elaboren incluso con ausencia datos y que pueden traspasar el ámbito de las reglas lingüísticas para hacer interferencias inteligentes en contextos donde sea complicado, o imposible, valerse exclusivamente de las herramientas del lenguaje (Artificial Solutions, 2020).

Un chatbot eficaz ofrece resultados ayudando a los pacientes a hacer algo sin necesidad de intervención humana. Adicionalmente, brinda una experiencia simple y de baja fricción (Jenkins et al., 2007). Así, este desarrollo digital transforma las alternativas en los procesos de comunicación y agiliza la entrega de información. 


\section{Influencia de los bots y aplicaciones en la comunicación de crisis}

Los desastres naturales y las pandemias no se pueden prever y plantean desafíos singulares a la prestación de servicios de salud; en consecuencia, la pandemia de Covid19 ha supuesto un gran trabajo tanto para investigadores como para desarrolladores tecnológicos, ya que equipos técnicos en todo el mundo han buscado soluciones digitales para ayudar a aliviar la presión sobre cuestiones médicas en los centros de salud.

Varias organizaciones gubernamentales y organismos de salud han utilizado plataformas de medios sociales como Facebook, WhatsApp, Instagram y Twitter para proporcionar información específica sobre Covid-19 a la población. WhatsApp es uno de los principales métodos para proporcionar información sobre la Covid-19 en muchos países (Hutchinson, 2020). Un ejemplo de ello son los gobiernos de Australia, India, Singapur y el Reino Unido.

La creación de todas las aplicaciones de Covid-19 ha servido para recopilar y registrar los datos relacionados con la salud del usuario. Las primeras que se han desarrollado y divulgado ampliamente han sido las aplicaciones de rastreo de contactos, que se crearon para notificar a sus usuarios si se habían cruzado con otra persona infectada con el coronavirus. La primera de ellas se hizo en Singapur (Baharudin, 2020). Después surgieron las aplicaciones para vigilar que los ciudadanos cumplieran el confinamiento, al igual que los asistentes conversacionales que contestaban las dudas frecuentes de la población.

Por su parte, las aplicaciones de vigilancia de los síntomas han sido útiles para identificar las tendencias de la enfermedad y las posibles zonas de alto contagio. La mayoría de los países hicieron las aplicaciones y los chatbots basándose en el manual de síntomas de la enfermedad y en los cuestionarios que los propios usuarios iban contestando (Hanson et al., 2020).

A continuación, se enlistan algunos de los bots que surgieron en España para sumarse a la lucha sanitaria en la pandemia correspondientes a las instituciones con las que se ha contactado para realizar este estudio. 
Tabla 1. Robots conversacionales en España.

\begin{tabular}{|c|c|c|c|}
\hline Empresa & Vía & Nombre chatbot & Institución \\
\hline 1. 1millionbot & web y WhatsApp & Carina Bot & $\begin{array}{c}\text { Ayuntamiento de } \\
\text { Alicante y Elche }\end{array}$ \\
\hline 2. Semanticbots & web & Chatbot Covid-19 & Once \\
\hline 3. Hubtype & WhatsApp y Telegram & gencatBot & Generalitat de Catalunya \\
\hline $\begin{array}{c}\text { 4. Chatbot Chocolate } \\
\text { 5. CARTO, } \\
\begin{array}{c}\text { FerceManager y } \\
\text { apoyo de Telefónica, } \\
\text { Ferrovial, Google y } \\
\text { Googo. }\end{array}\end{array}$ & web & Maia & Servicio Vasco de Salud \\
\hline
\end{tabular}

\section{Fuente: Elaboración propia.}

Carina ha sido desarrollada por 1Millionbot en España. Se trata de una plataforma conversacional gratuita sobre coronavirus basado en IA que utiliza fuentes oficiales como la Organización Mundial de la Salud (OMS) y fuentes oficiales gubernamentales. Esta tecnología, lanzada en la primera mitad de marzo de 2020, ha sido insertada por diferentes entidades en sus páginas web. Al igual que Carina en España, esta empresa desarrolló un mes después para la República de Ecuador, el chatbot Catalina. El Programa de las Naciones Unidas para el Desarrollo (PNUD) adjudicó a esta sociedad alicantina el desarrollo de Catalina para dar una respuesta urgente a las demandas de los ciudadanos de Ecuador. Este bot se aloja en 79 páginas oficiales, y en tres meses de funcionamiento tuvo una interacción de 135.000 usuarios (Torre Juana, 2020).

El equipo de Semanticbots está especializado en alta tecnología y puso a disposición gratuita de las asociaciones y organismos la iniciativa del chatbot Covid-19. Este asistente virtual, lanzado a mediados de abril de 2020, fue desarrollado utilizando la plataforma de SayOBO, la cual es accesible para diversos soportes y dispositivos. Además, el asistente virtual incluye consultas relacionadas con síntomas, medidas de higiene, medidas laborales y derechos sociales. Las respuestas y la base de conocimiento que posee el chatbot proceden de fuentes oficiales como es el caso de la Organización Mundial de la Salud (OMS) (Sayobo, 2019).

Hubtype ha creado, junto a la Consejería de Salud, un robot conversacional sobre el coronavirus para La Generalitat a través de aplicaciones de mensajería instantánea como WhatsApp. Esta compañía se autodefine como una empresa que "crea experiencias conversacionales de calidad" (Hubtype, 2020). El bot, disponible desde junio de 2020, comparte contenidos sobre salud y protección civil, así como datos de afectados, consejos, medidas para el confinamiento e informaciones sobre la evolución de la pandemia de forma gratuita. 
Chatbot Chocolate es "la agencia líder en el diseño, desarrollo y entrenamiento de chatbots y voice apps" (Chatbot Chocolate, 2020). Maia, asistente conversacional lanzado por esta empresa a principios de abril de 2020, es capaz de responder más de 125 preguntas diferentes formuladas en más de 50.000 formas distintas en Euskera y Castellano (Planeta Chatbot, 2020).

La Comunidad de Madrid optó por la creación de una plataforma inspirada en Corea del Sur que fue lanzada a finales de marzo de 2020. La función de CoronaMadrid es evaluar mediante una serie de preguntas la situación clínica del ciudadano. Por medio de esta autoevaluación básica, el usuario puede conocer la magnitud de sus síntomas y es orientado sobre el procedimiento a seguir en cada caso, pudiendo volver a realizarse la evaluación cada 12 horas para incorporar las novedades que hubiesen podido suceder en su evolución. Además, este sistema permite obtener datos epidemiológicos y ofrecer información personalizada, y para ofrecer las mejores medidas preventivas y de evaluación, incluye un sistema de geolocalización" (Pérez, 2020).

\section{Metodología}

Esta investigación presenta los resultados de un estudio cualitativo y cuantitativo. El primero está desarrollado a partir de una entrevista en profundidad realizada a cinco expertos representantes de las empresas anteriormente citadas, que se efectuó durante julio y agosto. El segundo es un cuestionario realizado en septiembre y octubre de preguntas cerradas a una muestra de 100 personas de entre 18 y 65 años en toda España. Asimismo, para completar el análisis, se realizó un trabajo de campo para obtener información de primera mano de los chatbots empleados. Este trabajo consistió en hacer una lista de preguntas a los asistentes comenzando por cuestiones más genéricas sobre información del virus para ver el nivel de comprensión e información, para pasar después a realizar otras sobre posibles efectos y consejos, buscando la recomendación de esta tecnología y consejos de telemedicina. Por estas razones, en los bots se observaron los siguientes parámetros:

- Políticas de privacidad.

- El saludo inicial

- Los idiomas que tiene.

- La batería de preguntas y respuestas que posee.

- Si da recomendaciones sobre la enfermedad.

- Si existe un diagnóstico.

- Información sobre los centros de salud.

La entrevista en profundidad que se define como una "técnica social que pone en relación de comunicación directa cara a cara a un investigador/entrevistador y a un individuo entrevistado con el cual se establece una relación peculiar de conocimiento que 
es dialógica, espontánea, concentrada y de intensidad variable" (Canales, 2006, p. 219). Esta produce información de carácter cualitativo, buscando generar mayor riqueza del material lingüístico recabado.

Para este estudio, la entrevista se diseñó en cuatro bloques. En el primero, se analizaron aspectos generales sobre el diseño de la herramienta: objetivos perseguidos, recursos, tiempo empleado y diseño del árbol de decisión. Después, se desarrolló una batería de cuestiones acerca de empresas y organismos que han empleado esta tecnología, y la estimación de dinero que estas se han ahorrado gracias a su uso. En el tercero, se preguntó sobre la cifra de usuarios, empleabilidad y manejo, para saber cuándo se han usado más por parte de los ciudadanos y, por último, se concluyó con una valoración del desarrollo virtual en cuestión y de su tecnología de cara al futuro. Gracias a estas entrevistas se pudo conocer la percepción de los expertos en cuanto a diseño y posibles líneas de mejora.

Por su parte, el objetivo de un cuestionario es medir el grado o la forma en que los sujetos encuestados poseen determinadas variables y conceptos de interés para la investigación. Se busca presentar al sujeto respuestas preestablecidas para facilitar el proceso de realización y posteriormente, de medición (Canales, 2006); por eso el cuestionario elaborado fue de respuestas cerradas. A través de las distintas preguntas se solicitaron datos sociodemográficos, de empleabilidad, sobre manejo y usabilidad, al igual que una valoración general de la herramienta utilizada durante la pandemia y posibles sugerencias para mejorarla. De esta forma se ha pretendido contrastar el punto de vista de los expertos con el del mercado a través de las valoraciones de los propios usuarios.

\section{Resultados}

\section{Trabajo de campo}

Las siguientes tablas recogen los resultados más relevantes del trabajo de campo realizado sobre los chatbots que se han utilizado en el estudio: 
Tabla 2. Comparativa robots conversacionales.

\begin{tabular}{|c|c|c|c|c|c|}
\hline & Carina Bot & Covid-19 & gencatBot & Maia & CoronaMadrid \\
\hline $\begin{array}{l}\text { Solicitud de número de } \\
\text { teléfono y política } \\
\text { privacidad }\end{array}$ & no & no & no & sí & sí \\
\hline $\begin{array}{l}\text { Informa sobre la } \\
\text { utilización datos }\end{array}$ & no & no & no & no & Sí \\
\hline Saludo inicial & sí & sí & sí & sí & sí \\
\hline Idioma & $\begin{array}{l}\text { Castellano e } \\
\text { inglés }\end{array}$ & $\begin{array}{l}\text { Castellano e } \\
\text { inglés }\end{array}$ & Catalán & $\begin{array}{l}\text { Castellano y } \\
\text { euskera }\end{array}$ & $\begin{array}{l}\text { Castellano, inglés, } \\
\text { francés y alemán. }\end{array}$ \\
\hline Preguntas & Predefinidas & Predefinidas & Predefinidas & Predefinidas & Guión cerrado \\
\hline Respuestas & Predefinidas & Predefinidas & Predefinidas & Predefinidas & sí/no \\
\hline Recomendación & sí & sí & sí & sí & sí \\
\hline Historial/perfil & sí & no & no & no & sí \\
\hline Diagnóstico & no & no & no & no & sí \\
\hline $\begin{array}{l}\text { Información sobre centros } \\
\text { sanitarios }\end{array}$ & no & no & no & no & sí \\
\hline
\end{tabular}

Fuente: Elaboración propia.

Tabla 3. Pregunta común entre los chatbots. ${ }^{4}$

\begin{tabular}{|l|l|l|l|l|l|}
\cline { 2 - 5 } \multicolumn{1}{c|}{} & Carina Bot & Covid-19 & gencatBot & Maia & CoronaMadrid \\
\hline $\begin{array}{l}\text { Tengo fiebre, tos } \\
\text { y dificultad } \\
\text { respiratoria }\end{array}$ & $\begin{array}{l}\text { Quédate en casa, No tengo } \\
\text { cuídate y vigila } \\
\text { tus síntomas }\end{array}$ & $\begin{array}{l}\text { Contacta con } \\
\text { tu centro de } \\
\text { atención primaria, } \\
\text { llama al 061 o haz } \\
\text { pregunta }\end{array}$ & $\begin{array}{l}\text { Llame } \\
\text { ina consulta a } \\
\text { través de Mi Salud } \\
\text { tal 900203050 }\end{array}$ & no estés bien \\
\hline
\end{tabular}

\section{Fuente: Elaboración propia.}

A continuación, se presentan los pantallazos del saludo inicial que cada uno tiene.

\footnotetext{
${ }^{4}$ Se hicieron las mismas preguntas en las cinco plataformas, aunque alguna de ellas como es el caso de Corona Madrid, solo permite ceñirse a un guion de cuestiones establecidas por el programa.
} 
Figura 2. Saludo de los bots.
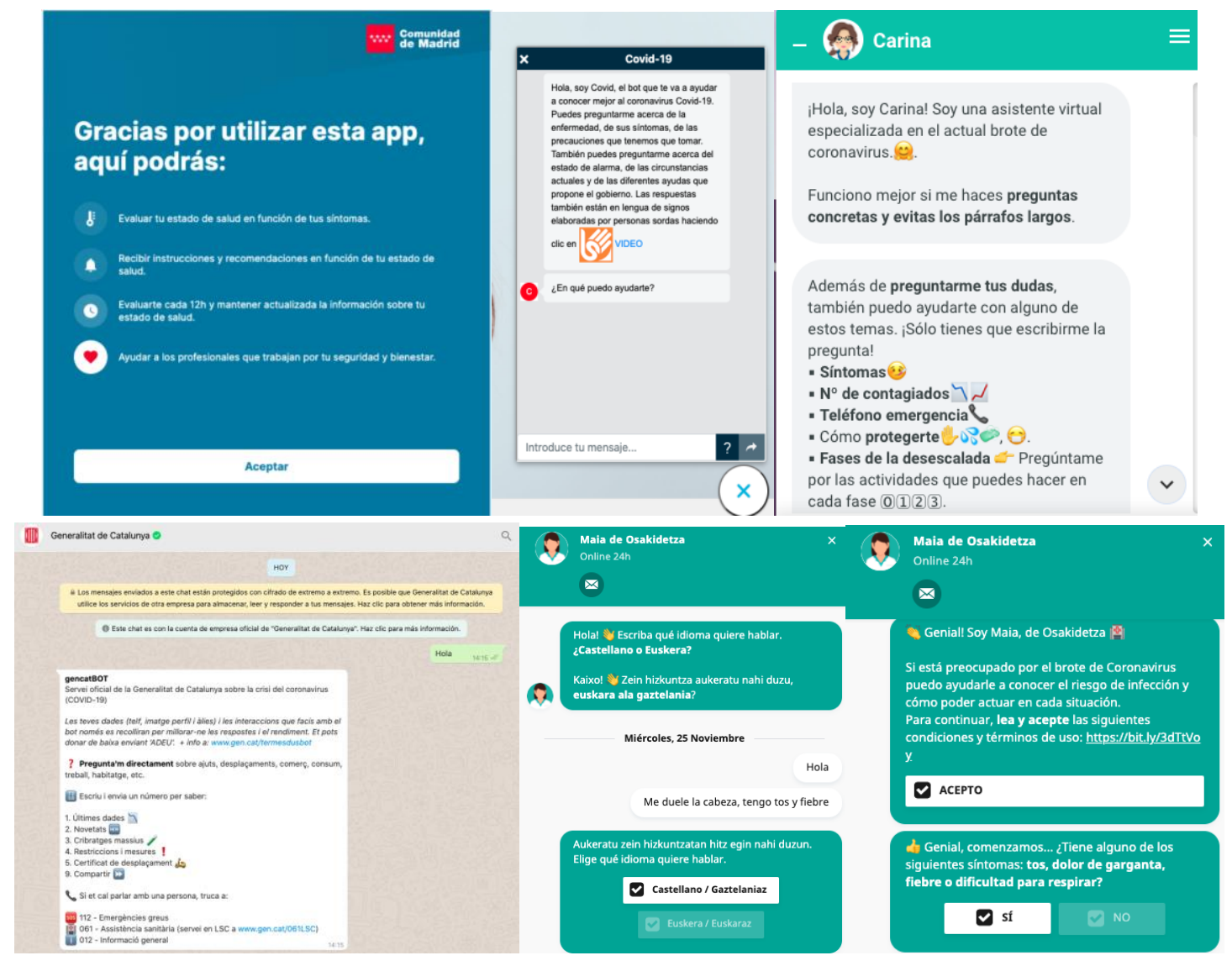

Fuente: Carina, CoronaMadrid, Maia, gencaBOT, 2020.

\section{Entrevistas a expertos}

En los siguientes párrafos se recogen los datos más significativos obtenidos a través de las entrevistas que, como se ha dicho anteriormente, fueron organizadas en torno a cuatro bloques de preguntas.

En el primer bloque, referido a cuestiones propias del diseño del bot, se ha observado una coincidencia entre todos los profesionales entrevistados en cuanto a que los chatbots han sido útiles en la crisis sanitaria. El 50\% valoraron la inmediatez, la facilidad de uso y la transparencia. El otro $50 \%$ se refirieron a la utilidad para descongestionar canales de comunicación y para proporcionar otros nuevos. Con respecto al tiempo empleado en el diseño, la mayoría lo estimó entre 4 y 8 semanas, y solo el $25 \%$ manifestó haber dedicado una única semana. Por último, el esfuerzo humano puesto al servicio del diseño ha sido importante, indicando que se contó con equipos de 8 y 4 personas, que, en la práctica, suponía utilizar sus recursos humanos al completo.

En el segundo bloque, se observaron respuestas más dispares en cuanto al número de empresas que han utilizado la tecnología. El 75\% dijo que menos de 10, frente al $25 \%$ que indicó más de 300. Respecto a la estimación del ahorro de recursos que ha podido 
suponer el hecho de que las empresas utilicen este tipo de plataformas, el 100\% manifestó la dificultad de estimar esta cifra o respondió de forma genérica diciendo que "muchos".

En el tercer bloque, en el que se estimaron los datos de uso, el 100\% coincidió en que los robots conversacionales han mantenido un uso constante, correspondiendo los momentos de mayor utilización con las primeras semanas del estado de alarma, debido a la situación de desinformación y confusión que se había generado. Respecto al cálculo de usuarios, sólo una empresa ha proporcionado una cifra concreta que es la de 120.000.

Por último, en el cuarto bloque, destinado a que los entrevistados realizaran la valoración general de la herramienta, la respuesta general ha sido óptima, en cuanto que todos ellos afirmaron que estos asistentes han permitido resolver muchas preguntas. Como puntos negativos, los expertos destacaron la necesidad de entrenamiento por parte de la herramienta y la serie de cuestiones que este tipo de bots es incapaz de resolver como sí lo haría un ser humano. Pero, a pesar de los defectos que estos sistemas puedan tener, y que ellos mismos han reconocido, se mostraron optimistas, considerando todos los elementos positivos que incorporan, y confiados en que se produzca una implantación generalizada de este tipo de tecnología. Uno de los entrevistados manifestó además, que, en su opinión, los cambios en nuestra vida cotidiana que esta pandemia ha traído consigo y los que aún acarreará harán que los chatbots supongan, cada vez más, una ayuda clave para los ciudadanos.

\section{Encuestas}

Teniendo en cuenta las variables sociodemográficas, son los jóvenes de 15-25 años los que más emplean este tipo de tecnología y el $40 \%$ proceden de Madrid, siendo el chatbot más empleado en el conjunto de la muestra el denominado CoronaMadrid. Más de la mitad de los encuestados aseguraron que utilizar este tipo de plataformas sí los ayudó durante la pandemia.

Figura 3. Porcentaje de respuestas ante la pregunta ¿le ayudó?

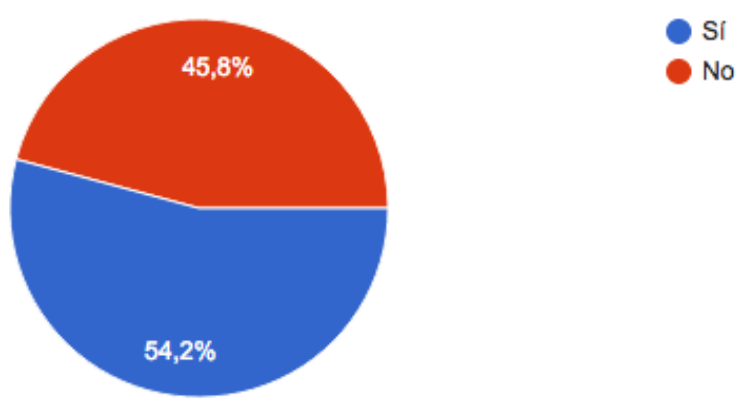

Fuente: Elaboración propia. 
El $45 \%$ afirmó que ha empleado esta herramienta con fines informativos, concretamente para identificar síntomas y saber qué hacer en caso de contagio y al hacer uso de sus opciones al $90 \%$ le pareció fácil su manejo. Respecto a los periodos durante los cuales utilizaron estos asistentes, la mayoría de los participantes respondieron que fue durante los meses de marzo, abril, mayo y septiembre, produciéndose una disminución de consultas en los meses estivales. No obstante, los picos más altos de uso se encontraron en los meses de abril y octubre. La curva de uso se aprecia en el siguiente gráfico:

Figura 4. Curva de uso de bots.

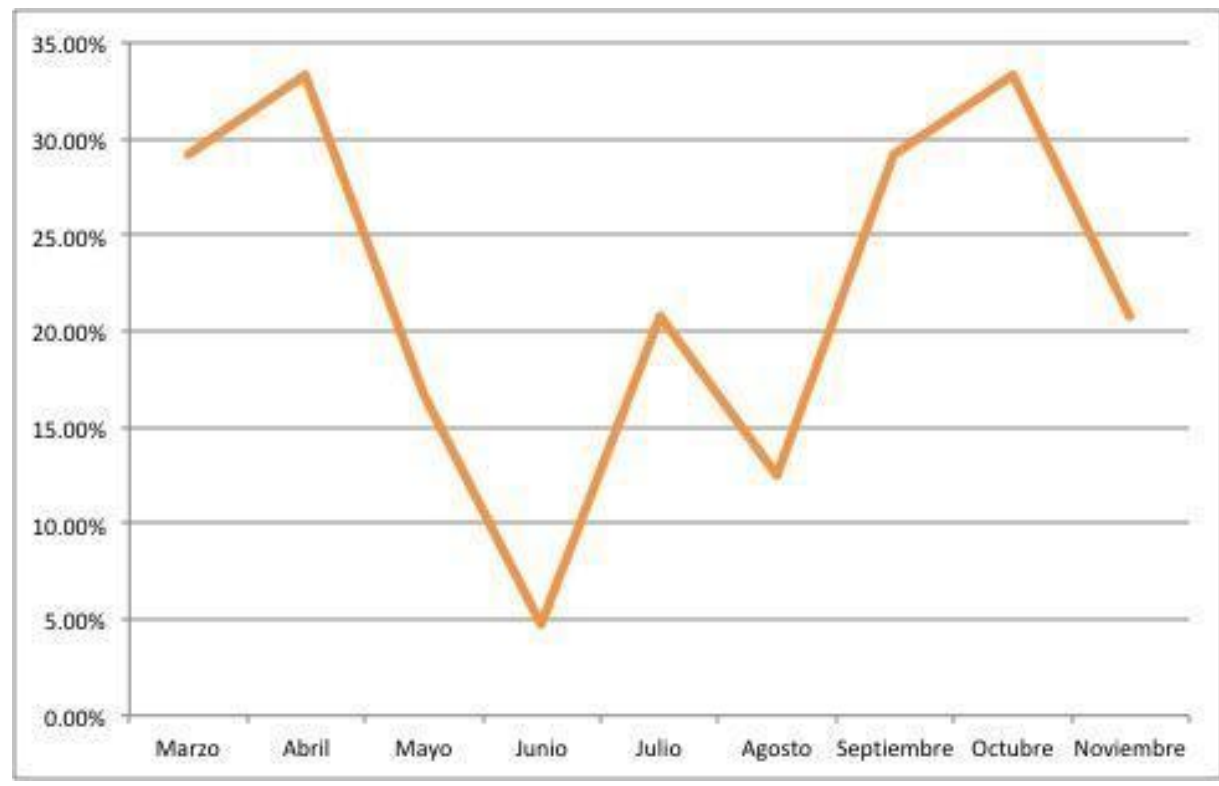

Fuente: Elaboración propia.

Al hablar de la utilidad general de la plataforma escogida, al 55\% le pareció útil y muy útil y un 37,5\% de los encuestados afirmó que no cambiaría nada para mejorarla. Además, el 29\% aseguró que utilizar este sistema evitó que tuviera que contactar con su centro de salud ya que la consulta realizada lo dejó satisfecho.

Por último, casi un 33\% de los encuestados han usado esta tecnología durante estos meses de pandemia y el resto no ha utilizado ninguna aplicación específica sobre salud. Las personas que no consideraron útiles estos servicios son las que pertenecen a la franja de edad de 46-55 años y más de 56.

\section{Discusión y Conclusiones}

La entrevista realizada ha sido fundamental para conocer las opiniones de los profesionales que están detrás del desarrollo de esta tecnología. Los resultados de este estudio proporcionan una instantánea de la importancia atribuida a los chatbots en esta crisis sanitaria y lo primero que se deduce, una vez analizadas las respuestas, es el carácter informativo que tienen la mayoría de estos robots conversacionales además de que todos los entrevistados inciden en la importancia de su labor durante las primeras semanas del estado de alarma. Se ha detectado a través de este análisis que los sistemas incorporan un 
grado bajo de Inteligencia Artificial, especialmente al tratarse del uso de lenguaje natural en la interacción comunicativa entre chatbot- usuario; en varios de los casos sucedió que el bot no entendía las preguntas realizadas, lo cual significa que las opciones en el flujo de conversación son limitadas y poco variadas.

Otra conclusión derivada de la entrevista en profundidad realizada a los expertos y protagonistas del diseño de estas plataformas es que casi la totalidad de ellos, un $96 \%$, se mostraron satisfechos con el cumplimento de los objetivos que se habían propuesto en su diseño, concretamente en los de ser una herramienta informativa fiable y que colaborara a crear nuevos canales comunicativos que consiguieran descongestionar los tradicionales. Sin embargo, como reflejan las encuestas realizadas a los usuarios, sólo un $29 \%$ de los participantes afirmó que no contactó con su centro de salud después de realizar las consultas a través de estos medios. Y es que, a pesar de que estas herramientas fueron diseñadas para descongestionar un sistema sanitario que comenzaba a colapsarse, la realidad es que no han evitado de forma significativa que la gente acudiera a sus centros de salud, pues estas herramientas -todavía se encuentran en una primera fase de desarrollo en cuanto a Inteligencia Artificial (IA)-, no están preparadas para responder a determinadas cuestiones complejas o a hacerlo con la seguridad que proporciona al usuario el contacto persona a persona a la hora de explicar en detalle sus problemas.

En el análisis realizado respecto a las empresas desarrolladoras, se advierte una gran improvisación y ausencia de medición de resultados. No se ha estimado el alcance, la repercusión, ni la eficacia de uso de sus productos. Aunque esta deficiencia puede ser fruto de lo reciente de la implantación de este tipo de tecnologías en la lucha contra la Covid-19, lo cierto es que sobre estas cuestiones solo una empresa facilitó datos de mercado.

Asimismo, los cuestionarios han ayudado a completar el estudio y a tener la perspectiva de experiencia de uso de los ciudadanos y, en este sentido, queda claro el motivo principal por el que los encuestados han acudido a estas plataformas y es la búsqueda de información. Concretamente, se producen dos picos de utilización correspondientes a los meses de abril y octubre, que coinciden además con las dos olas de contagios en España por lo que se puede deducir que el empeoramiento de las cifras de contagios y muertes está relacionado con la demanda de acceso a este tipo de fuentes de información.

La información proporcionada por la muestra respecto a la plataforma más utilizada sitúa a CoronaMadrid en primer lugar, dato que está en concordancia con la procedencia de la mayoría de los encuestados, aunque otros manifestaron no recordar el nombre del chatbot utilizado. Llama la atención que algunas personas señalaran a RadarCovid como plataforma utilizada, ya que esta es una app que requiere de descarga y que está destinada fundamentalmente a registrar a las personas contagiadas y a avisar a los ususarios de la posibilidad de haber estado en contacto con algún positivo, pero no se trata de un bot. Esto apunta al desconocimiento en cuanto a la diferenciación de una u otra tecnología por parte de los usuarios y a qué servicios ofrece cada una. En la 
actualidad gran parte de los ciudadanos se instalan diferentes aplicaciones en sus teléfonos móviles para uso de entretenimiento, profesional, e incluso educativo, pero una de las cosas que distingue precisamente a los robots conversacionales del resto de apps es que estos no necesitan descarga, ya que funcionan en línea a través de webs o de Redes Sociales.

Gracias al trabajo de campo se ha observado que todos los chatbots analizados coinciden en varias de sus características. De hecho, dos de las plataformas estudiadas, y que no pertenecen a la misma empresa, tienen un look $\&$ feel muy similar (color verde y blanco: Carina y Maia) coincidiendo también, en este caso, en la elección de un nombre e imagen femenina para personalizar a su asistente conversacional.

Figura 5. Robots conversacionales con apariencia de mujer.

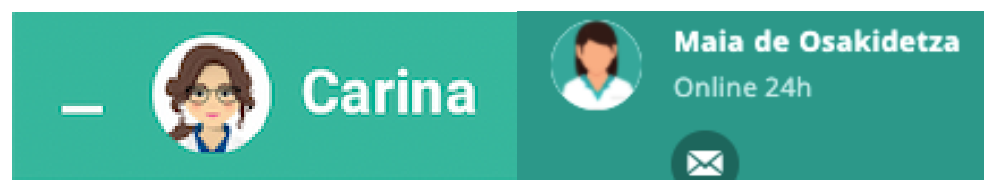

Fuente: Carinarina y Maia, 2020.

A este respecto es interesante mencionar que el pasado año, la Unesco, a través de la publicación del informe I'd blush if I could (Me sonrojaría si pudiera), cuyo título alude a la respuesta dada por Siri cuando los usuarios la insultaban de forma soez, recomendó tener presente la perspectiva de género en el desarrollo de las competencias digitales para no perpetuar estereotipos femeninos como la sumisión, la sensualidad o el servilismo. El resto de plataformas analizadas, sin embargo, cuentan con un asistente que no está basado en la virtualización de un ser humano. Por otro lado, la primera interacción que ofrecen todas estas plataformas es un saludo de bienvenida, pero solo algunas informan, antes de utilizar el servicio, de los objetivos de su creación o el uso que se hará de los datos proporcionados. Y, aunque dos de los bots estudiados pertenecen a sistemas de salud institucionales, solo uno monitoriza (a través de criterios de geolocalización) el estado del ciudadano y cruza los datos con el sistema sanitario correspondiente, lo que parece una tarea sencilla, ya que solo con un número de teléfono el asistente es capaz de identificarnos y comenzará a dirigirse a nosotros por nuestros apellidos. Este hecho nos inclina a pensar que estas plataformas tienen acceso a un historial de consultas previas realizadas utilizando el mismo teléfono como medio de identificación. Por último, respecto al estudio de caso, se puede decir que en cuanto a la accesibilidad solamente una empresa: Semanticbots es incluyente, pues su diseño está adaptado para personas con problemas de visión.

El confinamiento - decretado en marzo de 2020, como consecuencia del estado de alarma- aceleró la adopción de esta tecnología en el área de la atención médica. En estos meses los bots han mejorado; por ejemplo, han incluido: recomendaciones, información de perfil y datos para contactar con los centros sanitarios. No obstante, siguen atendiendo a un árbol de respuestas y una de sus limitaciones más claras es que solo responden a ciertas preguntas predefinidas. 
Figura 6. Test en CoronaMadrid.

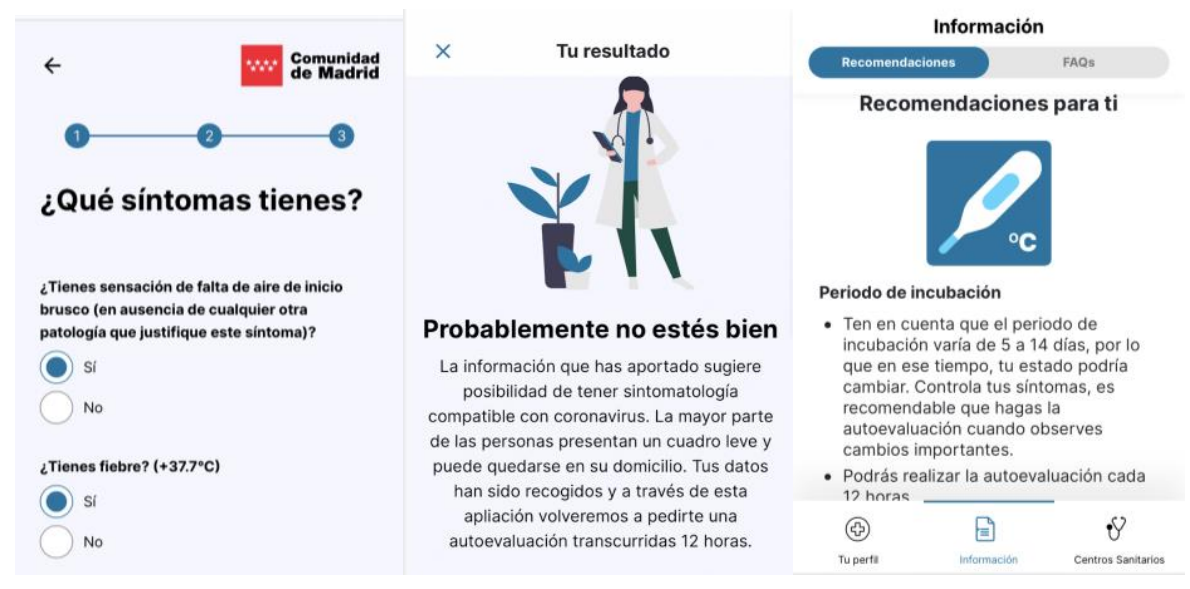

Fuente: CoronaMadrid, 2020.

Se puede concluir diciendo que todas estas plataformas convencionales han colaborado a difundir las medidas preventivas que establecieron las autoridades sanitarias como la difusión del famoso "Quédate en casa", recomendación que todas ellas incluyen. Además suponen, gracias al tipo de lenguaje utilizado y a la estructuración de las respuestas programadas, una herramienta de refuerzo que enfatiza los mensajes respecto a cómo actuar en caso de contagio y, al mantener un tono informativo, consiguen también transmitir una sensación tranquilizadora, evitando que los usuarios entren en pánico y puedan actuar de forma caótica y poco efectiva para sus intereses y los del resto de los ciudadanos.

Figura 7. Recomendación realizada por los bots.

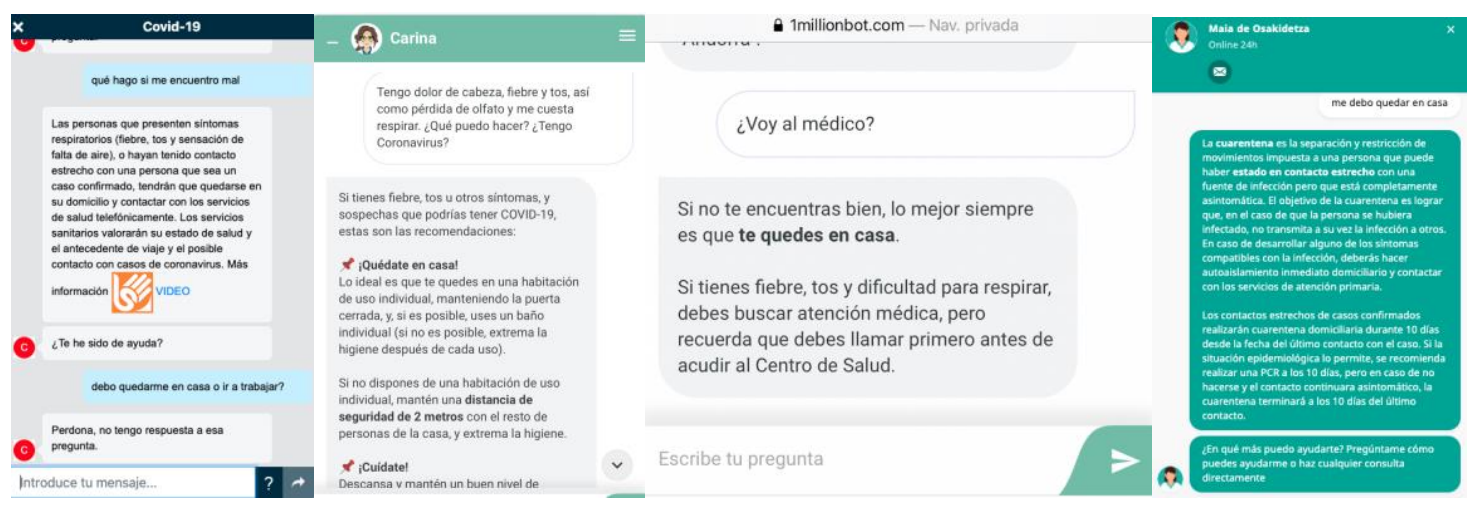

Fuente: Covid-19, Carina y Maia, 2020.

Con estos desarrollos tecnológicos se impulsa un camino en los proyectos de automatización de flujos, especialmente dentro del sector sanitario, ya que gracias a la experiencia de usuario y recolección de datos, existe una constante mejora en el rendimiento del software. A largo plazo, cuando se termine esta crisis, es probable que los chatbots se conviertan en portales digitales de atención sanitaria interactiva, ayudando a los pacientes y médicos y facilitando, de este modo, la primera atención en una emergencia de salud. Por lo tanto, el seguimiento del desarrollo e implantación de los 
robots conversacionales en el área sanitaria y su aplicación en situaciones de crisis sigue siendo un campo todavía muy novedoso que, sin duda, invita a los investigadores a mantenerse atentos y a abrir nuevas vías de investigación.

\section{Bibliografía}

Ahuja, A., Reddy, V. y Marques, O. (2020). Artificial Intelligence and COVID-19: A Multidisciplinary Approach, Integrative Medicine Research, 9(3) doi.org/10.1016/j.imr.2020.100434.

Aguilar, J y Villegas, O. (2020). COVID-19 y sus implicaciones sociales: una mirada desde las ciencias computacionales. https://tinyurl.com/y58k32x2

Artificial Solutions. Chatbots: la guía definitiva (2020). https://www.artificialsolutions.com/es/chatbots-pdf/

Baharudin, H. (2020). Coronavirus: Singapore Government to make its contact-tracing app freely available to developers worldwide. https://tinyurl.com/yyz7ldwk

Bird, D., Ling, M., y Haynes, K. (2012). Flooding Facebook -the use of social media during the Queensland and Victorian floods, Australian Journal of Emergency Management, 27(1), 27-33.

Brandtzaeg P., Folstad A. (2017). Why People Use Chatbots. Internet Science. doi.org/10.1007/978-3-319-70284-1_30.

Canales, M. (2006). Metodologías de investigación social. Chile: Lom Ediciones.

Dahiya, M. (2017). A Tool of Conversation: Chatbot, International Journal of Computer Sciences and Engineering, 5(5).

Ferrara, B; Varol, O; Davis, C; Menczer, F y Flammini, A. (2016). The Rise of Social Bots, Communications of the Acm, 59(7).

Flores, D. (09 de mayo de 2020). España alcanzó el pico de la epidemia el 26 de marzo, once días después de declarar el estado de alarma. RTVE/Coronavirus. https://www.rtve.es/noticias/20200509/espana-alcanzo-pico-epidemia-26marzo-once-dias-despues-declarar-estado-alarma/2013735.shtml

Freshdesk. (2020). How to Improve Chatbot Conversation Strategy. https://freshdesk.com/self-service-portal/improve-chatbot-conversation-blog/

Grimme, C., Preuss, M., Adam, L., y Trautmann, H. (2017). Social Bots: Human-Like by Means of Human Control?, Big Data, 5(4), 279-293, doi: 10.1089/big.2017.0044. 
Hanson, J., Couch, D., y Yap, K. (2020). Mobile Health Apps That Help With COVID19 Management: Scoping Review. JMIR Nursing. 3(1). https://www.nursing.jmir.org/2020/1/e20596/\#ref75.

Herriman, M., Meer E., Rosin R., Lee V., Washington V., y Volpp, K. (2020). Asked and Answered: Building a Chatbot to Address Covid-19-Related Concerns, NEJM Catalyst. doi: 10.1056/CAT.20.0230.

Hofeditz, L., Ehnis, C., Bunker, D., Brachten, F., y Stieglitz, S. (2019). Meaningful use of social bots? Possible applications in crisis communication during disasters. En Proceedings of the 27th European Conference on Information Systems (ECIS), Stockholm y Uppsala, Suecia, Junio 8-14, 2019. ISBN978-1-7336325-0-8 Research Papers. https://aisel.aisnet.org/ecis2019_rp/138.

Hubtype. (2020). Mensajería escalable. https://www.hubtype.com/es/.

Hutchinson, A. (2020). WhatsApp launches new COVID-19 info hub amid concerns around misinformation being shared in-app. https://tinyurl.com/y6hj3pll.

Jenkins, M., Churchill R., Cox S., y Smith D. (2007) Analysis of User Interaction with Service Oriented Chatbot Systems. Computer Science, 4552. Doi: 10.1007/9783-540-73110-8_9.

Kunze, L. (2016). On chatbots. https://techcrunch.com/2016/02/16/on-chatbots/.

Lind, M., y Salomonson. N. (2006). The Role of Virtual Servants in e-Interaction. https://tinyurl.com/y2ybrnhv.

Malpica, J. (2016). Inteligencia artificial y consciencia. Universidad Alcalá de Henares. http: //www.3.uah.es.

Miner, A., Laranjo, L., y Kocaballi, A. (2020). Chatbots in the fight against the covid-19 pandemic, Npj Digital Medicine, 3(1). doi.org/10.1038/s41746-020-0280-0.

Moncloa. (2020, 10 de abril). La Generalitat crea un 'bot' que da información sobre el Covid-19 por WhatsApp. https://tinyurl.com/y3cvb645.

Mori, M. (1970). The Uncanny Valley. Energy, 7(4), 33-35. Doi:10.1109/MRA.2012.2192811

Organización Panamericana de la Salud y Organización Mundial de la Salud. (26-30 de septiembre de 2011). Estrategia y plan de acción sobre eSALUD. 51. ${ }^{\circ}$ Consejo Directivo. https://www.paho.org/hq/dmdocuments/2011/cd51-13-s.pdf.

Pérez, J. (2020, 18 de marzo). Madrid lanza una web para atender a posibles afectados de coronavirus. https://tinyurl.com/y3f4tvxm.

Planeta Chatbot. (2020, 28 de abril). Tecnología de chatbot Chocolate en Osakidetza. https://tinyurl.com/y3mxxbdn. 
Prendinger, H. y Ishizuka, M. (2004). Life-like characters: Tools, affective functions, and applications. Springer Science \& Business Media.

Sánchez-Gonzales, Hada M.; Sánchez-González, María (2017). Los bots como servicio de noticias y de conectividad emocional con las audiencias. El caso de Politibot. Doxa comunicación, 25, 63-84.

Sayobo. (2019). Chatbot COVID- 19. www.sayobo.io.

Shawar, A. y Atwell, E. (2005). Using corpora in machine-learning chatbot systems, International Journal of Corpus Linguistics, 10(4), 489-516.

Stieglitz, S., Bunker, D., Mirbabaie, M. y Ehnis, C. (2017). Sense-Making in Social Media During Extreme Events, Journal of Contingencies and Crisis Management, 26(1), 4-15.

Torre Juana. (2020, 25 de julio). Naciones Unidas y 'Catalina': ayudando más allá de los síntomas de COVID-19. https://tinyurl.com/y4voya46.

Trillo-Domínguez, Magdalena; Alberich-Pascual, Jordi (2017). Deconstrucción de los géneros periodísticos y nuevos medios: de la pirámide invertida al cubo de Rubik. El profesional de la información, 26(6),1091-1099. doi.org/10.3145/epi.2017.nov.08

Van den Broek, E., Zarouali, B., y Poels, K. (2019). Chatbot advertising effectiveness: When does the message get through?, Computers in human behaviour, 98. doi.org/10.1016/j.chb.2019.04.009.

Van Eijck, M. y Claxton, N.X. (2009). Rethinking the notion of technology in education: Techno-epistemology as a feature inherent to human praxis. Science Education, 93(2), pp. 218-232.

Wilks, Y. (2010). Close Engagements with Artificial Companions. Key social, psychological, ethical and design issues. Holanda: John Benjamins.

World Health Organization. (2020, 12 de marzo). WHO announces COVID-19 outbreak a pandemic. https://tinyurl.com/y48wdsed.

Zarouali, B., Van den Broeck, E., Walrave, M., y Poels, K. (2018). Predicting consumer responses to a chatbot on Facebook, Cyberpsychology, Behavior, and Social Networking, 21(8), 491-497. https://doi.org/10.1089/cyber.2017.0518. 